\title{
Nonisentropic Release of a Shocked Solid
}

\author{
P. G. Heighway, ${ }^{1}$ M. Sliwa, ${ }^{1}$ D. McGonegle, ${ }^{1}$ C. Wehrenberg, ${ }^{2}$ C. A. Bolme, ${ }^{3}$ J. Eggert, ${ }^{2}$ A. Higginbotham, ${ }^{4}$ \\ A. Lazicki, ${ }^{2}$ H. J. Lee, ${ }^{5}$ B. Nagler, ${ }^{5}$ H.-S. Park, ${ }^{2}$ R. E. Rudd, ${ }^{2}$ R. F. Smith, ${ }^{2}$ M. J. Suggit, ${ }^{1}$ D. Swift, ${ }^{2}$ F. Tavella, ${ }^{5}$ \\ B. A. Remington, ${ }^{2}$ and J. S. Wark $\circledast^{1, *}$ \\ ${ }^{1}$ Department of Physics, Clarendon Laboratory, University of Oxford, Parks Road, Oxford OX1 3PU, United Kingdom \\ ${ }^{2}$ Lawrence Livermore National Laboratory, P.O. Box 808, Livermore, California 94550, USA \\ ${ }^{3}$ Los Alamos National Laboratory, Bikini Atoll Road, SM-30, Los Alamos, New Mexico 87545, USA \\ ${ }^{4}$ York Plasma Institute, University of York, Heslington, York YO10 5DD, United Kingdom \\ ${ }^{5}$ SLAC National Accelerator Laboratory, 2575 Sand Hill Road, Menlo Park, California 94025, USA
}

(Received 18 May 2019; revised manuscript received 9 September 2019; published 13 December 2019)

\begin{abstract}
We present molecular dynamics simulations of shock and release in micron-scale tantalum crystals that exhibit postbreakout temperatures far exceeding those expected under the standard assumption of isentropic release. We show via an energy-budget analysis that this is due to plastic-work heating from material strength that largely counters thermoelastic cooling. The simulations are corroborated by experiments where the release temperatures of laser-shocked tantalum foils are deduced from their thermal strains via in situ $\mathrm{x}$-ray diffraction and are found to be close to those behind the shock.
\end{abstract}

DOI: 10.1103/PhysRevLett.123.245501

Shock release is the fundamental thermodynamic process that takes place when a material at high pressure undergoes rapid decompression. Release of this sort occurs when a shock wave traveling through a solid inevitably reaches a free surface and thus encounters an unconfined boundary where the normal stress must vanish. The ensuing rarefaction wave sent back into the sample allows it to release from the state of high stress imposed by the rapid shock compression. It is currently accepted that this relatively slow release process is isentropic: this view is espoused by many textbooks on shock physics [1-4], and it is still extensively employed by those interpreting freesurface velocity histories [5-10] and release-melting pressures [11-14] to interrogate material behavior at extreme pressures and strain rates. Since the temperature of an isentropically expanding material decreases monotonically with volume due to the thermoelastic effect [15] (at a rate dictated by the Grüneisen parameter $\gamma$ [16]), a sample that releases from hundreds of gigapascals is assumed to cool by several hundred degrees.

However, the above picture fails to account for the fact that, although the strain rates present in the rarefaction fan are indeed significantly lower than those induced by the shock - and reduce as the fan propagates - within the first few microns of the surface, they can easily exceed $10^{9} \mathrm{~s}^{-1}$. Laser-compression studies [17-19] and molecular dynamics (MD) simulations performed on commensurate timescales [20-22] have shown that, in such an ultrahigh strain rate regime $\left(\dot{\varepsilon}^{p}>10^{7} \mathrm{~s}^{-1}\right)$, the solid will typically exhibit strength of order gigapascals. The considerable strength will therefore cause plastic-work heating not only during compression but also upon release (as shown in computational works by Swift et al. [23] and Kurosawa and Genda [24]), directly opposing the cooling due to expansion and causing the release path to deviate from the isentrope.

Moreover, an isentropic treatment of release necessarily ignores the energy content of the defects mediating plastic flow. It has long been posited that defects must be created in huge numbers during shock compression to accommodate the extreme plastic strain rates at the shock front $[25,26]$, in accordance with Orowan's equation [27]. This prediction is supported by MD simulations, which show copious homogeneous defect generation across the shock front $[21,28,29]$. Such simulations have also shown that these large defect densities can be partially annihilated upon release [30,31], and it has recently been demonstrated by in situ femtosecond x-ray diffraction (XRD) measurements that both deformation twinning and lattice rotation induced by shock compression of tantalum [32] are largely reversed during rarefaction [31], consistent with such a defect annihilation. The energy stored by the defects (which may constitute $5 \%$ to $15 \%$ of the plastic work performed on compression [33-38]) can thus be partially recovered during rarefaction and released as thermal energy, providing another source of heat that further opposes thermoelastic cooling.

It is within the above context that we present direct evidence of heating of a crystal upon shock release to temperatures greatly exceeding those expected from an isentropic expansion. We observe this effect in MD simulations of shock and rarefaction waves in single-crystal tantalum, and we show via an energy-budget analysis that the release path departs from the isentrope due to 
plastic-work heating and defect annihilation. We consider the depth dependence of the release temperature and show that, over the micron-scale of targets frequently used in laser-induced shock experiments, the temperature is fairly uniform. We confirm these predictions with experiments that provide temperature measurements via in situ XRD recordings of the thermally-induced expansive strains of laser-shocked tantalum foils. Our results challenge the current consensus that shock release is isentropic.

We performed large-scale MD simulations using the LAMMPS code [39] to simulate the shock and subsequent release of $300 \mathrm{~K}, 24.8 \times 24.8 \times 1120 \mathrm{~nm}^{3}$ tantalum monocrystals subjected to periodic boundary conditions (PBCs) on their transverse faces and loaded along their [011] axis to $100 \mathrm{GPa}$ with a constant-velocity piston. The crystals were modeled under the Ravelo EAM Ta2 potential [40], which was chosen in part for its reasonable description of tantalum's thermal properties (see the Supplemental Material [41]). We analyze a set of Lagrangian material elements within the rear $0.3 \mu \mathrm{m}$ of fully released material, each of which spans 30 lattice spacings $(10 \mathrm{~nm})$ along the loading axis. By examining the stress, strain, and microstructural changes effected in these elements by the release process, we can quantify the heating and cooling mechanisms governing their temperature.

In Fig. 1(a), we plot the temperature $T$ and elastic components of strain $\varepsilon^{e}$ of an element found $0.2 \mu \mathrm{m}$ from the rear surface, and in Fig. 1(b) we plot its dislocation
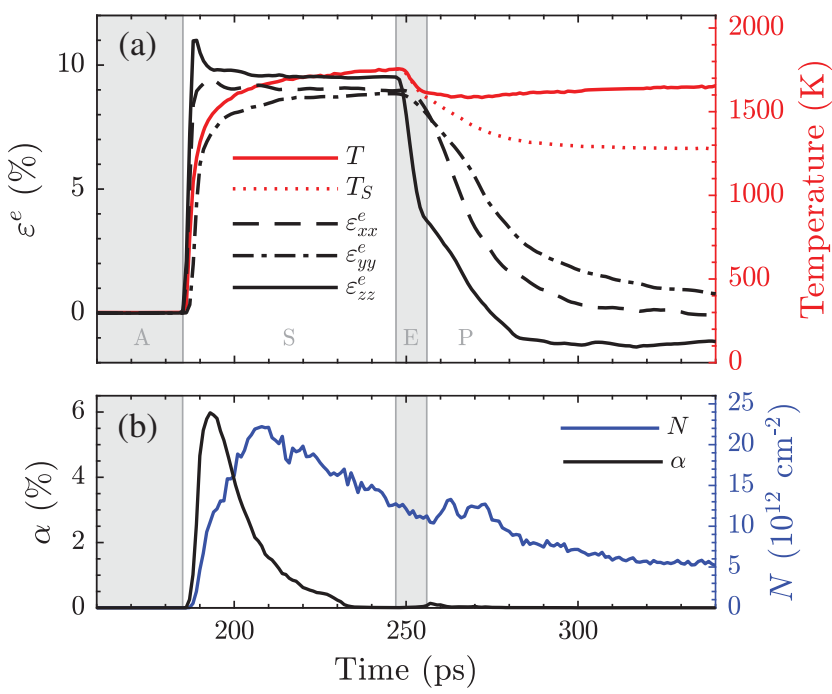

FIG. 1. Time evolution of a 10-nm-thick Lagrangian element of material initially situated $200 \mathrm{~nm}$ below the rear surface of a single crystal shock compressed along $z$ to $100 \mathrm{GPa}$. (a) Temperature $T$ and true elastic strain $\varepsilon^{e}$. Shown also is $T_{s}$, the temperature along the release isentrope emanating from the shock state at $t=247 \mathrm{ps}$. (b) Dislocation density $N$ (calculated using the dislocation extraction algorithm [58,59]) and twin fraction $\alpha$. Regions $A, S, E$, and $P$ denote when the crystal was under ambient conditions, shock compressed, elastically releasing, and plastically releasing, respectively. density $N$ and twin fraction $\alpha$. It can be seen that the passage of the shock wave at $185 \mathrm{ps}$ first heats the element to $1750 \mathrm{~K}$, and it generates deformation twins that are rapidly replaced by a dislocation network whose density peaks at $2.3 \times 10^{13} \mathrm{~cm}^{-2}$ (in fair agreement with densities inferred from experiments on [001] tantalum crystals [57]). The element suffers compression for $62 \mathrm{ps}$, during which time the shear stresses induced in it by the shock relax to a limiting value of $0.9 \mathrm{GPa}$ [with the corresponding shear strain $\Delta \varepsilon^{e}=\varepsilon_{z z}^{e}-\left(\varepsilon_{x x}^{e}+\varepsilon_{y y}^{e}\right) / 2$ falling to just $0.6 \%$ ], and the dislocation network steadily decreases in density while it equilibrates. Meanwhile, the shock traverses the remaining $200 \mathrm{~nm}$ of the crystal, breaks out from the rear surface, and launches a rarefaction wave back into the sample that encounters the element at $247 \mathrm{ps}$. The ensuing release takes place in two phases.

Initially, the element expands rapidly along the loading axis, but it suffers little change $(<0.5 \%)$ to its strains orthogonal to the shock. The conditions of lateral confinement (enforced by PBCs) require that macroscopic plastic flow cannot take place without changes to the transverse elastic strains $\varepsilon_{x x}^{e}$ and $\varepsilon_{y y}^{e}$. We thus deduce that the first phase of expansion is elastic and therefore proceeds isentropically. Indeed, the attendant temperature drop of $123 \mathrm{~K}$ as the longitudinal elastic strain $\varepsilon_{z z}^{e}$ decreases from $9.5 \%$ to $4.3 \%$ is largely consistent with the thermoelastic cooling rate expected within the Grüneisen formalism. This reads $\dot{T}_{\mathrm{TE}}=T \gamma: \dot{\varepsilon}^{e}$, where : is the double inner product operator, and $\gamma_{i j}(V)=V\left(\partial \sigma_{i j} / \partial E\right)_{V}\left(\approx 1.5 \delta_{i j}\right.$ at $\left.100 \mathrm{GPa}\right)$. Integration of this cooling rate yields a temperature drop of $130 \mathrm{~K}$, so we can predict the effect of cooling due to expansion reasonably well.

The elastic period of release ceases at 254 ps when the shear strain $\Delta \varepsilon^{e}$ reaches $4.2 \%$, and the corresponding shear stress is sufficiently large that the crystal yields. The subsequent plastic flow, evinced by reduction of the transverse elastic strains, deposits substantial thermal energy into the material via plastic work. Simultaneously, the dislocation density jumps by $25 \%$ to accommodate the high plastic strain rate of order $4 \times 10^{9} \mathrm{~s}^{-1}$, as shown in Fig. 1(b). Following a plateau of $10 \mathrm{ps}$ duration, the dislocations partially annihilate, decreasing in density from 13 to $5 \times 10^{12} \mathrm{~cm}^{-2}$. The potential energy recovered from the diminishing dislocations provides additional thermal energy that continues to heat the element at late times. By quantifying the heating from plastic work and recovery of defect energy during this plastic phase of release, we can give a reasonable prediction of the element's temperature profile.

Plastic work is performed at the rate $\dot{W}^{p}=\sigma: \dot{\varepsilon}^{p}$ per unit volume, where $\varepsilon^{p}$ is the plastic strain. For uniaxial loading, $\dot{\varepsilon}^{p}$ can be deduced from the elastic strain rate $\dot{\varepsilon}^{e}$ and the plastic dilatation rate $\dot{\varepsilon}_{V}^{p} \equiv \operatorname{Tr} \dot{\varepsilon}^{p}$ :

$\dot{W}^{p}=\left(\sigma_{x x}-\sigma_{z z}\right)\left(-\dot{\varepsilon}_{x x}^{e}\right)+\left(\sigma_{y y}-\sigma_{z z}\right)\left(-\dot{\varepsilon}_{y y}^{e}\right)-\sigma_{z z}\left(-\dot{\varepsilon}_{V}^{p}\right)$. 
A brief discussion of the origin of the (often neglected) dilatation may be found in the Supplemental Material [41]. If this work were completely converted to heat, the heating rate would read $\dot{T}_{\mathrm{PW}}=\dot{W}^{p} / c_{V}$, where $c_{V}$ is the element's volumetric heat capacity. This heating alone would be substantial given the huge shear stresses $\left(\sigma_{x x, y y}-\sigma_{z z}\right) / 2 \approx$ $3 \mathrm{GPa}$ present in the release fan. In reality, thermal energy density is deposited at a rate $\dot{W}^{p}-\dot{E}^{s} / V$, where $E^{s}$ is the energy stored by defects. We encode this correction with the term $\dot{T}_{\mathrm{MS}}=-\dot{E}^{s} /\left(V c_{V}\right)$ :

$$
\begin{aligned}
\dot{T} & =\dot{T}_{\mathrm{TE}}+\dot{T}_{\mathrm{PW}}+\dot{T}_{\mathrm{MS}} \\
& =T \gamma: \dot{\varepsilon}^{e}+\frac{1}{c_{V}}\left(\dot{W}^{p}-\dot{E}^{s} / V\right) .
\end{aligned}
$$

We thus account for additional heating owing to the thermal energy released by annihilation of dislocations, whose typical energy per unit length is $2 \mathrm{eV}^{-1}$. We now compare the temperature evolution predicted by this equation (a detailed discussion of which can be found in the Supplemental Material [41]) with the true release path.

In Fig. 2(a), we plot the heating rates resulting from plastic work and from defect energy recovery according to Eq. (2b) alongside the thermoelastic cooling rate, and in Fig. 2(b) we compare the release paths inferred from these rates with the observed release path. It can be seen that plastic work provides the bulk of the heating that counteracts the thermoelastic effect, and so largely compensates for the cooling effect of expansion. Inclusion of the smaller microstructural contribution, meanwhile, allows us to reproduce the shape of the release path with greater fidelity: in particular, we capture the slow temperature rise after $275 \mathrm{ps}$, during which the energy stored by the diminishing
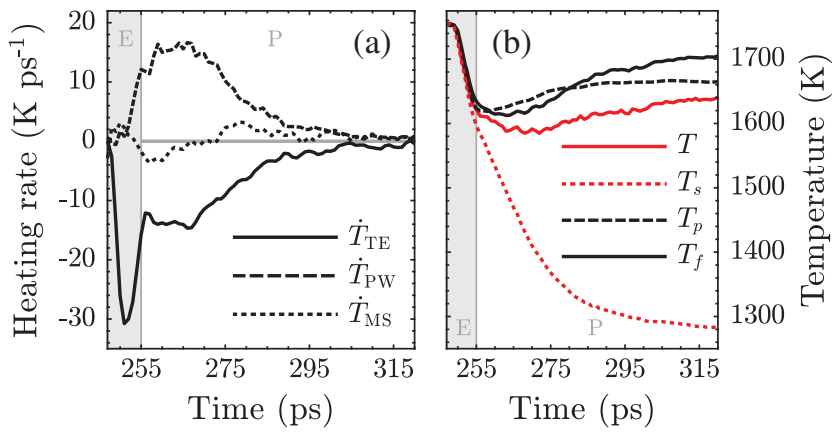

FIG. 2. Thermal-energy-budget analysis for a material element $200 \mathrm{~nm}$ below the free surface of a monocrystal releasing from $100 \mathrm{GPa}$ to ambient pressure. (a) Heating rates resulting from the thermoelastic effect $\left(\dot{T}_{\mathrm{TE}}\right)$, plastic work $\left(\dot{T}_{\mathrm{PW}}\right)$, and recovery of microstructure energy $\left(\dot{T}_{\mathrm{MS}}\right)$. (b) Comparison of the release path $T$ and corresponding isentrope $T_{s}$ with paths predicted from the various heating rates; $T_{f}$ contains the correction from $\dot{T}_{\mathrm{MS}}$, whereas $T_{p}$ does not. Periods of elastic and plastic release are labeled $E$ and $P$, respectively. dislocation network is gradually released as heat. There does exist a discrepancy between the measured and predicted temperatures $T$ and $T_{f}$, which we attribute to our application of the Grüneisen formalism (an equilibrium thermodynamics concept) to a system in which large numbers of nonequilibrium defects (dislocations) are being nucleated and annihilated. However, the difference is sufficiently small that we conclude the release heating mechanism is indeed a combination of plastic work and recovery of defect energy.

We further note that the release temperature varies with distance from the rear surface of the crystal. Since the top of the release fan propagates faster than its tail, it broadens over time, causing the local rarefaction rate to fall with increasing distance from the free surface. Lower strain rates can be accommodated by slower-moving dislocations, which can in turn be driven by smaller shear stresses, as we show for a series of Lagrangian elements in Fig. 3(a). The local plastic work performed therefore decreases with depth [see Fig. 3(b)]. Since plastic work provides the bulk of the heating, we expect material further into the sample to be cooler. We verify this in Fig. 3(c), where we show the temperature variation over $3 \mu \mathrm{m}$ of fully released material obtained from the simulation of a larger crystal some $6 \mu \mathrm{m}$ long. However, the length scale over which the release temperature decays is of order microns: the average temperature within the last $3 \mu \mathrm{m}$ of released material is $1520 \mathrm{~K}$-still some $250 \mathrm{~K}$ above the isentrope. We note
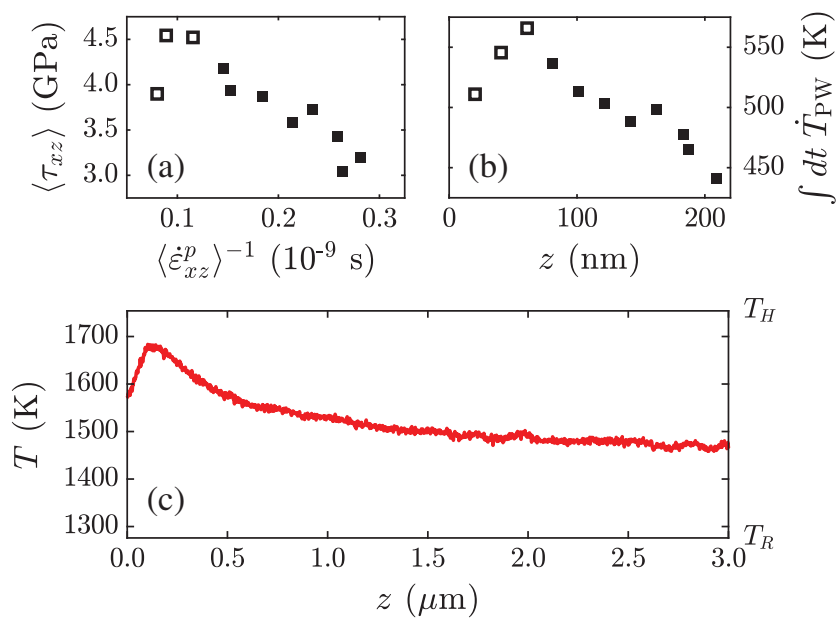

FIG. 3. (a) Time-averaged shear stress $\left\langle\tau_{x z}\right\rangle$ on planes at $45^{\circ}$ to the loading direction against inverse plastic shear strain rate $\left\langle\dot{\varepsilon}_{x z}^{p}\right\rangle^{-1}$ for a set of material elements releasing from a $100 \mathrm{GPa}$ shock. (b) Plastic-work heating versus distance from the rear surface z. (c) Temperature profile for a 6- $\mu \mathrm{m}$-long crystal unloading from $100 \mathrm{GPa}$ at $1.3 \mathrm{~ns}$ after breakout. $T_{H}$ and $T_{R}$ denote the shock and isentropic release temperatures, respectively. The finite timescale of shock heating means that material nearest the rear surface never reaches the shock temperature and is thus cooler; elements in this region are marked by hollow points. 
that the thermal diffusion time (which MD overestimates due to omission of electron conductivity) for a micron-scale temperature gradient in a tantalum crystal with known thermal diffusivity $D \approx 23 \mathrm{~mm}^{2} \mathrm{~s}^{-1}$ [60] is of order $100 \mathrm{~ns}$. Hence while the excess temperature at the rear surface might diffuse into the cooler bulk over the microsecond duration of the typical gas-gun experiment, it certainly could not do so during the nanosecond timescale of a lasercompression experiment using micron-scale foils. We will now describe the results of just such an experiment, in which we observe in situ the nonisentropic release of tantalum foils and thus confirm the predictions of our simulations.

Experiments were undertaken at the Matter in Extreme Conditions (MEC) end station of the Linac Coherent Light Source (LCLS) [61]. The setup is identical to that shown in the previous work of Wehrenberg et al. [32] and Sliwa et al. [31]; we recap the essential details here. The targets were $6-\mu \mathrm{m}$-thick foils of polycrystalline $\mathrm{Ta}$ with a fiber texture such that the majority of the crystallites were oriented with their [011] axis parallel, within a few degrees, to the target normal, but around this axis the grains were oriented approximately randomly. A $50-\mu \mathrm{m}$-thick polyimide ablator was glued to the front surface of the metallic foils. A frequency-doubled (527-nm) laser containing energies between 5 and $25 \mathrm{~J}$ in a 5 - to 10-ns pulse was focused onto the ablator. Phase plates were used to produce focal spots of 100,150 , or $250 \mu \mathrm{m}$ diameter. Shock pressures were deduced from measurements of the velocity of the foil's rear surface by use of a velocity interferometer system for any reflector (VISAR) $[62,63]$.

XRD patterns of the foils under conditions of shock and release were obtained by illuminating them with $9.6-\mathrm{keV}$ $\mathrm{x}$ rays of $50 \mathrm{fs}$ duration incident at $35^{\circ}$ to their surface normal. Owing to the fiber-textured nature of the targets, this results in arcs of intense diffracted signal being recorded on the detector, rather than full Debye-Scherrer rings. The $\mathrm{x}$ rays were focused to a spot of $20 \mu \mathrm{m}$ diameter (FWHM) aligned to the center of the spot produced by the optical laser. While the majority of the $\mathrm{x}$ rays were contained within this spot, scattering in the Be lenses that focus the $\mathrm{x}$ rays causes a weak "halo" of $\mathrm{x}$ rays to be focused to a diameter larger than the optical focal spot. This halo thus always diffracts from unshocked material, giving a reference position of diffraction angle from which the tensile strain after shock breakout can be deduced. The time at which the $\mathrm{x}$ rays scatter from the target relative to breakout time was monitored using the streak camera recording the VISAR signal.

Typical XRD data for a shock pressure of $130 \mathrm{GPa}$ during shock and release are shown in Fig. 4(a). Shortly before breakout, the $\mathrm{x}$-ray beam is most strongly scattered an angle $2 \theta$ that exceeds the ambient scattering angle due to lattice compression. As the crystal rarefies during the passage of the release fan, its lattice spacings increase, (a)
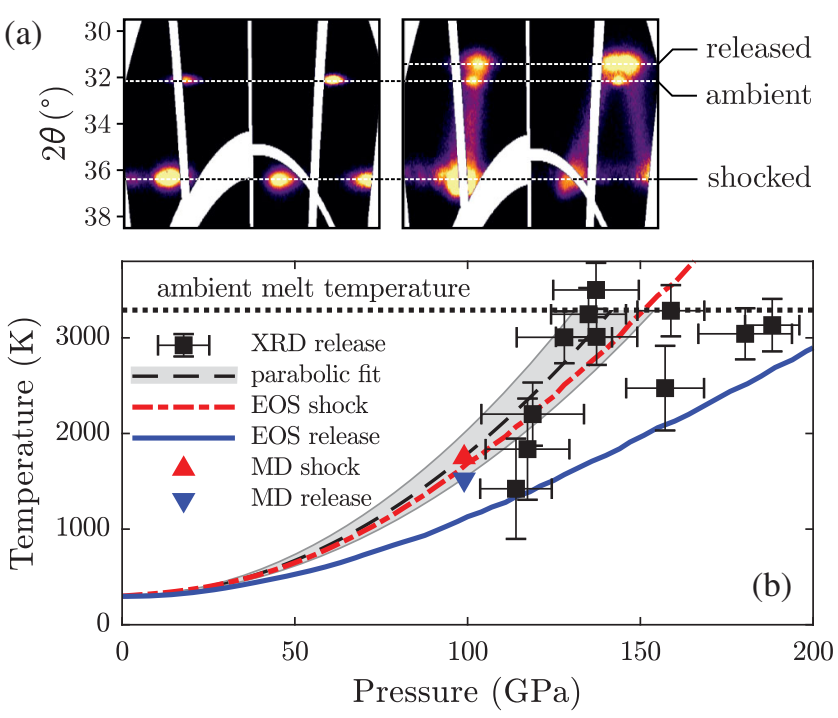

FIG. 4. (a) Images of the $\{011\}$ diffraction peaks from crystals loaded to $130 \mathrm{GPa}$ (left panel) before and (right panel) after breakout. (b) Release temperature inferred from XRD as a function of shock pressure $p$ less than $1.2 \mathrm{~ns}$ after breakout. The data for which $p<150 \mathrm{GPa}$ are fitted to a parabola whose $95 \%$ confidence interval is shaded gray. Shown also are the shock and corresponding isentropic release temperatures predicted from SESAME equation of state (EOS) 3520 [64], and temperatures from a $6-\mu \mathrm{m}$-long MD simulation.

causing the diffraction intensity to shift back to lower values of $2 \theta$. The angle to which the peaks return once rarefaction is complete is lower than that under ambient conditions. Since the free-surface boundary condition forces the normal stress $\sigma_{z z}$ of the released material to fall to zero, the expansion we observe relative to the ambient state must be interpreted as thermal expansion. As explained in the Supplemental Material [41], this allows us to deduce from the tensile elastic strains the temperature of the released crystal using the known thermal expansion coefficient of tantalum $\left(\approx 6.5 \times 10^{-6} \mathrm{~K}^{-1}\right)$ [60].

For shocks with strength of order $100 \mathrm{GPa}$, the typical release temperature is indeed substantially higher than one would expect were the release to be isentropic. In Fig. 4(b), we show the release temperature inferred from XRD as a function of shock pressure for samples probed at the latest $\Delta t=1.2 \mathrm{~ns}$ after shock breakout-within this time, at most $C_{0} \Delta t \approx 4 \mu \mathrm{m}$ of material has been completely released (where $C_{0}$ is the ambient bulk sound speed $[65,66])$; hence a direct comparison with our $6-\mu \mathrm{m}$ release simulation is warranted. In all cases, the inferred release temperature exceeds the theoretical isentropic release temperature calculated using a high-pressure equation of state [64]. In fact, the data appear to follow more closely the Hugoniot (the locus of shock states) up to around $150 \mathrm{GPa}$, above which the release temperature saturates at the ambient melt temperature $T_{M}=3270 \mathrm{~K}$ [67] (due to the finite enthalpy of melting preventing the release path from 
crossing the melt curve). We calculated the $95 \%$ confidence interval of one-parameter parabolic fits to the unsaturated data of the form $T(p \mid \kappa)=300 \mathrm{~K}+\kappa p^{2}$ using Bayesian regression, and we found that the interval excludes the locus of isentropic release states. We therefore assert that the experimental data and the MD simulations are consistent: both indicate that the uniaxial shock release of micron-scale tantalum from hundreds of gigapascals is a highly nonisentropic process.

Finally, we should stress that this release heating phenomenon is not peculiar to tantalum. From Eq. (2b), we see that the ratio of $\dot{T}_{\mathrm{PW}}$ to $\dot{T}_{\mathrm{TE}}$ for a material element for which both $\gamma$ and $\dot{\varepsilon}^{e}$ are scalar is

$$
R=\frac{4}{3}\left(\frac{\tau}{c_{V} T}\right) \frac{1}{\gamma}
$$

where $\tau$ is the shear stress. Any material for which this dimensionless "figure of merit" $R$ approaches or exceeds unity will release nonisentropically.

In summary, we have performed MD simulations of shock release in tantalum and have found that its temperature greatly exceeds that expected from an isentropic release. This is due in part to release of the energy stored by the defects, but it is dominated by plastic-work heating owing to material strength. The simulations are consistent with experiments where thermally induced strains of lasershocked targets were recorded via femtosecond x-ray diffraction. These results challenge the textbook understanding of release and illustrate the importance of combined MD and experimental studies of lattice response under high strain rate, which can now be performed on comparable length scales and timescales.

We would like to thank S. M. Vinko and M. F. Kasim for their assistance in analyzing the experimental errors. J.S. W. is grateful to the UK EPSRC for support under Grants No. EP/J017256/1 and No. EP/S025065/1. M. S. and D.M. were supported by Lawrence Livermore National Security under Contracts No. B609694 and No. B595954, respectively, and D. M. acknowledges further support from AWE Aldermaston. The use of LCLS, SLAC National Accelerator Laboratory, is supported by the U.S. Department of Energy, Office of Science, Office of Basic Energy Sciences, under Contract No. DE-AC0276SF00515. The MEC instrument is supported by the U.S. Department of Energy, Office of Science, Office of Fusion Energy Sciences, under Contract No. SF00515. This material is based upon work supported by the U.S. Department of Energy, Office of Science, Office of Fusion Energy Sciences, under Award No. DE-SCW-1507. This work was performed in part under the auspices of the U.S. Department of Energy by Lawrence Livermore National Laboratory under Contract No. DE-AC52-07NA27344. *justin.wark@physics.ox.ac.uk

[1] Y. B. Zel'dovich and Y.P. Raizer, Physics of Shock Waves and High-Temperature Hydrodynamic Phenomena (Academic Press, New York, 1966).

[2] M. A. Meyers, Dynamic Behavior of Materials (John Wiley \& Sons, New York, 1994).

[3] J. W. Forbes, Shock Wave Compression of Condensed Matter (Springer, Berlin, 2012).

[4] L. Davison, Fundamentals of Shock Wave Propagation in Solids (Springer, Berlin, 2008).

[5] J. M. Walsh and R. H. Christian, Phys. Rev. 97, 1544 (1955).

[6] J. R. Asay, J. Appl. Phys. 46, 197 (1975).

[7] M. D. Knudson, J. R. Asay, and C. Deeney, J. Appl. Phys. 97, 073514 (2005).

[8] M. D. Knudson and M. P. Desjarlais, Phys. Rev. B 88, 184107 (2013).

[9] D. V. Minakov, P. R. Levashov, K. V. Khishchenko, and V. E. Fortov, J. Appl. Phys. 115, 223512 (2014).

[10] M. D. Knudson, M. P. Desjarlais, and A. Pribram-Jones, Phys. Rev. B 91, 224105 (2015).

[11] C. Dai, J. Hu, and H. Tan, J. Appl. Phys. 106, 043519 (2009).

[12] G. I. Kanel, J. Baumung, D. Rush, J. Singer, S. V. Razorenov, and A. V. Utkin, AIP Conf. Proc. 429, 155 (1998).

[13] T. Ye, Y. Yu-Ying, D. Cheng-Da, Y. Ji-Dong, W. Qing-Song, and T. Hua, Acta Phys. Sin. 62, 036401 (2013).

[14] M. G. Gorman, R. Briggs, E. E. McBride, A. Higginbotham, B. Arnold, J. H. Eggert, D. E. Fratanduono, E. Galtier, A. E. Lazicki, H. J. Lee, H. P. Liermann, B. Nagler, A. Rothkirch, R. F. Smith, D. C. Swift, G. W. Collins, J. S. Wark, and M. I. McMahon, Phys. Rev. Lett. 115, 095701 (2015).

[15] W. Thomson, Trans. R. Soc. Edinburgh 20, 261 (1853).

[16] A. M. Molodets, Combust., Explos. Shock Waves (Engl. Transl.) 37, 455 (2001).

[17] M. A. Meyers, F. Gregori, B. K. Kad, M. S. Schneider, D. H. Kalantar, B. A. Remington, G. Ravichandran, T. Boehly, and J. S. Wark, Acta Mater. 51, 1211 (2003).

[18] W. J. Murphy, A. Higginbotham, G. Kimminau, B. Barbrel, E. M. Bringa, J. Hawreliak, R. Kodama, M. Koenig, W. McBarron, M. A. Meyers, B. Nagler, N. Ozaki, N. Park, B. Remington, S. Rothman, S. M. Vinko, T. Whitcher, and J. S. Wark, J. Phys. Condens. Matter 22, 065404 (2010).

[19] A. J. Comley et al., Phys. Rev. Lett. 110, 115501 (2013).

[20] E. M. Bringa, A. Caro, Y. Wang, M. Victoria, J. M. McNaney, B. A. Remington, R. F. Smith, B. R. Torralva, and H. Van Swygenhoven, Science 309, 1838 (2005).

[21] E. M. Bringa, K. Rosolankova, R. E. Rudd, B. A. Remington, J. S. Wark, M. Duchaineau, D. H. Kalantar, J. Hawreliak, and J. Belak, Nat. Mater. 5, 805 (2006).

[22] R. E. Rudd, Mater. Sci. Forum 633-634, 3 (2009).

[23] D. C. Swift, A. Seifter, D. B. Holtkamp, and D. A. Clark, Phys. Rev. B 76, 054122 (2007).

[24] K. Kurosawa and H. Genda, Geophys. Res. Lett. 45, 620 (2018).

[25] C. S. Smith, Trans. Metall. Soc. AIME 212, 574 (1958).

[26] M. A. Meyers, Scr. Metall. 12, 21 (1978).

[27] E. Orowan, Proc. Phys. Soc. London 52, 8 (1940). 
[28] B. L. Holian and P. S. Lomdahl, Science 280, 2085 (1998).

[29] T. C. Germann, B. L. Holian, P. S. Lomdahl, and R. Ravelo, Phys. Rev. Lett. 84, 5351 (2000).

[30] H. N. Jarmakani, E. M. Bringa, P. Erhart, B. A. Remington, Y. M. Wang, N. Q. Vo, and M. A. Meyers, Acta Mater. 56, 5584 (2008).

[31] M. Sliwa, D. McGonegle, C. Wehrenberg, C. A. Bolme, P. G. Heighway, A. Higginbotham, A. Lazicki, H. J. Lee, B. Nagler, H. S. Park, R. E. Rudd, M. J. Suggit, D. Swift, F. Tavella, L. Zepeda-Ruiz, B. A. Remington, and J. S. Wark, Phys. Rev. Lett. 120, 265502 (2018).

[32] C.E. Wehrenberg, D. McGonegle, C. Bolme, A. Higginbotham, A. Lazicki, H. J. Lee, B. Nagler, H.-S. Park, B. A. Remington, R. E. Rudd, M. Sliwa, M. Suggit, D. Swift, F. Tavella, L. Zepeda-Ruiz, and J. S. Wark, Nature (London) 550, 496 (2017).

[33] W. S. Farren and G. I. Taylor, Proc. R. Soc. A 107, 422 (1925).

[34] G. I. Taylor and H. Quinney, Proc. R. Soc. A 143, 307 (1934).

[35] P. Rosakis, A. Rosakis, G. Ravichandran, and J. Hodowany, J. Mech. Phys. Solids 48, 581 (2000).

[36] J. Mason, A. Rosakis, and G. Ravichandran, Mech. Mater. 17, 135 (1994).

[37] M. Bever and L. Ticknor, Acta Metall. 1, 116 (1953).

[38] M. Bever, D. Holt, and A. Titchener, Prog. Mater. Sci. 17, 5 (1973).

[39] S. Plimpton, J. Comput. Phys. 117, 1 (1995).

[40] R. Ravelo, T. C. Germann, O. Guerrero, Q. An, and B. L. Holian, Phys. Rev. B 88, 134101 (2013).

[41] See Supplemental Material at http://link.aps.org/ supplemental/10.1103/PhysRevLett.123.245501, which includes Refs. [42-56], for discussions of the fidelity of the interatomic potential, the testing and implementation of the heat equation, and the routine used to extract release temperatures from the diffraction data.

[42] H. Cynn and C.-S. Yoo, Phys. Rev. B 59, 8526 (1999).

[43] A. Dewaele, P. Loubeyre, and M. Mezouar, Phys. Rev. B 70, 094112 (2004).

[44] A. C. Mitchell and W. J. Nellis, J. Appl. Phys. 52, 3363 (1981).

[45] A. Dewaele, M. Mezouar, N. Guignot, and P. Loubeyre, Phys. Rev. Lett. 104, 255701 (2010).
[46] J. M. Walsh, M. H. Rice, R. G. McQueen, and F. L. Yarger, Phys. Rev. 108, 196 (1957).

[47] R. E. Cohen and O. Gülseren, Phys. Rev. B 63, 224101 (2001).

[48] Z. Liu, L.-C. Cai, X.-R. Chen, Q. Wu, and F.-Q. Jing, J. Phys. Condens. Matter 21, 095408 (2009).

[49] J. Clayton, J. Mech. Phys. Solids 53, 261 (2005).

[50] P. Longère and A. Dragon, J. Theor. Appl. Mech. 45 (2007).

[51] C. H Rycroft, Chaos 19, 041111 (2009).

[52] R. Ravelo, B. L. Holian, and T. C. Germann, AIP Conf. Proc. 1195, 825 (2009).

[53] A. Higginbotham and D. McGonegle, J. Appl. Phys. 115, 174906 (2014).

[54] W. L. Stewart, J. M. Roberts, N. G. Alexandropolous, and K. Salama, J. Appl. Phys. 48, 75 (1977).

[55] K. Wang and R. R. Reeber, Mater. Sci. Eng. R 23, 101 (1998).

[56] Y. M. Gupta and S. J. Turneaure, J. Appl. Phys. 106, 033513 (2009).

[57] C. E. Wehrenberg, A. J. Comley, N. R. Barton, F. Coppari, D. Fratanduono, C. M. Huntington, B. R. Maddox, H. S. Park, C. Plechaty, S. T. Prisbrey, B. A. Remington, and R. E. Rudd, Phys. Rev. B 92, 104305 (2015).

[58] A. Stukowski and K. Albe, Model. Simul. Mater. Sci. Eng. 18, 085001 (2010).

[59] A. Stukowski, V. V. Bulatov, and A. Arsenlis, Model. Simul. Mater. Sci. Eng. 20, 085007 (2012).

[60] ASM Ready Reference: Thermal Properties of Metals, edited by F. Cverna (ASM International, Materials Park, $\mathrm{OH}, 2002)$.

[61] B. Nagler et al., J. Synchrotron Radiat. 22, 520 (2015).

[62] L. Barker and R. Hollenbach, J. Appl. Phys. 43, 4669 (1972).

[63] P. M. Celliers, D. K. Bradley, G. W. Collins, D. G. Hicks, T. R. Boehly, and W. J. Armstrong, Rev. Sci. Instrum. 75, 4916 (2004).

[64] B. Bennett, Sandia National Laboratories Report No. SCRR-70-28, 1983.

[65] R. G. McQueen, J. W. Hopson, and J. N. Fritz, Rev. Sci. Instrum. 53, 245 (1982).

[66] D. Orlikowski, P. Söderlind, and J. A. Moriarty, Phys. Rev. B 74, 054109 (2006).

[67] L. Malter and D. B. Langmuir, Phys. Rev. 55, 743 (1939). 